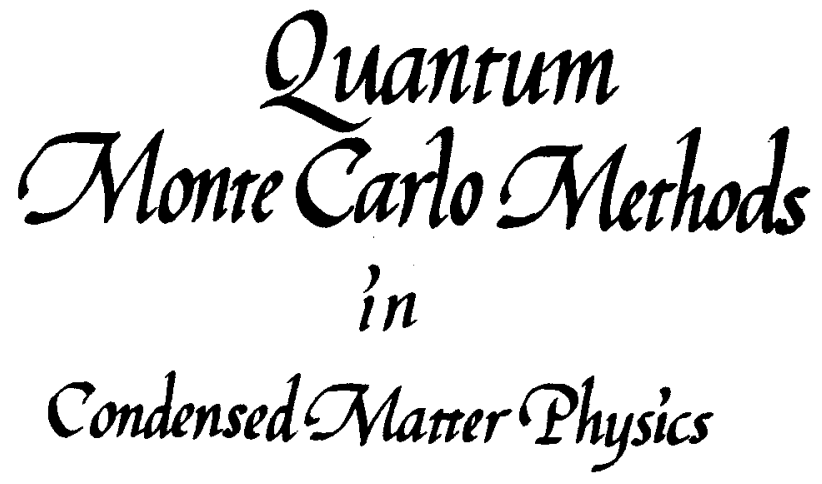


This page is intentionally left blank 


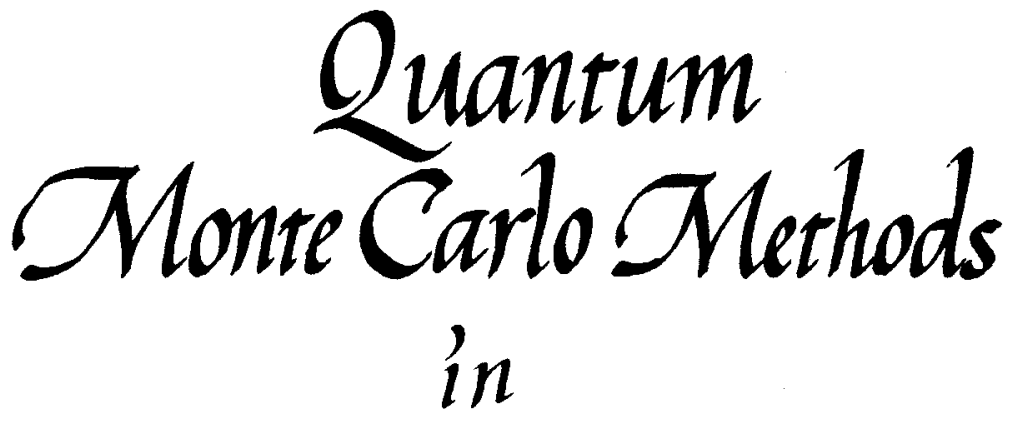

\section{Condensed Matter Physics}

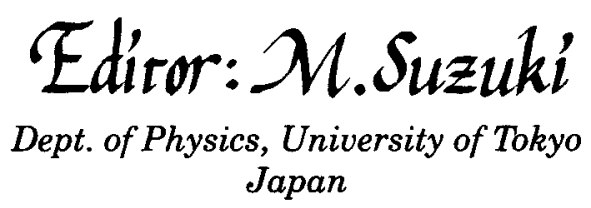




\section{Published by}

Word Scientific Publishing Co. Pte. Ltd.

P O Box 128, Fanrer Road, Singapore 9128

USA office: Suite 1B, 1060 Main Street, River Edge, NJ 07661

UK office: 73 Lynton Mead, Totteridge, London N20 8DH

\section{QUANTUM MONTE CARLO METHODS IN \\ CONDENSED MATTER PHYSICS}

Copyright $\odot 1993$ by World Scientific Publishing Co. Pte. Ltd.

All rights reserved. This book, or parts thereof, may not be reproduced in any form or by any means, electronic or mechanical, including photocopying, recording or any information storage and retrieval system now known or to be invented, without written permission from the Publisher.

For photocopying of material in this volume, please pay a copying fee through the Copyright Clearance Center, Inc., 27 Congress Street, Salem, MA 01970, USA.

ISBN 981-02-1659-9

Printed in Singapore. 


\section{PREFACE}

This book reviews recent developments of quantum Monte Carlo methods and some remarkable applications to quantum spin, fermion and boson systems.

The quantum Monte Carlo method is a very powerful and non-perturbational tool for studying thermal and quantal fluctuations in interacting quantum spin systems and strongly correlated electron systems. In the standard quantum Monte Carlo method based on the generalized Trotter formula, quantum fluctuations are expressed in terms of configurations in the additional Trotter dimension of the transformed system. This corresponds to the Feynman path integral. The path-summation scheme in quantum Monte Carlo simulations is particularly useful when a fairly large amount of machine time of high-speed computers is available.

This book contains twenty-two papers by thirty authors. The first paper gives the foundations of the standard quantum Monte Carlo method, including some recent results on higher-order decompositions of exponential operators and ordered exponentials. The second paper presents a general review of quantum Monte Carlo methods used in the present book. Some related methods such as the transfer-matrix method and the decoupled-cell method are also briefly explained. The negative-sign problem is also discussed. This is one of the most challenging problems in the field of quantum Monte Carlo techniques, as is also discussed in some other papers of the present book. Some new methods are proposed to overcome partially this difficult negative-sign problem.

Low-dimensional quantum spin systems are studied by several authors using quantum Monte Carlo methods or the quantum transfer-matrix method based on the equivalence theorem that a $d$-dimensional quantum system is equivalent to the corresponding $(d+1)$-dimensional classical system. Some new techniques such as the loop algorithm (namely a new type of cluster algorithm) are reported here to overcome critical slowing down in quantum Monte Carlo simulations. Random fields and inhomogeneity effects are studied in quantum spin systems. The critical exponents of quantum spin chains are also evaluated numerically using the quantum transfermatrix method. The Haldane gap is confirmed for spin $S=1$ and 2 by numerical calculations.

The variational Monte Carlo method is also included here together with some recent applications of it to the Hubbard model, the $t-J$ model and the Kondo lattice.

Some interesting applications of quantum Monte Carlo methods to fermion systems are also presented to investigate the role of strong correlations and fluctuations of electrons and to clarify the mechanism of the high- $T_{c}$ superconductivity. Not only thermal properties but also quantum-mechanical ground-state properties have been 
studied by the projection technique using auxiliary fields. The algorithm in the infinite dimensional limit is very useful to solve models of strongly correlated systems and to clarify a transition to an incommensurate magnetic state in the Hubbard model. The Hubbard-Stratonovich transformation and the auxiliary-fields approach are also effectively used to interpret geometrically the negative-sign problem in terms of a Berry phase in the Hubbard and Heisenberg models. The maximum entropy method is applied to the degenerate single-impurity Anderson model. Many other interesting topics on fermion and boson systems are discussed in the present book.

Numerical calculations of transport coefficients and relaxation functions derived by Kubo's linear-response theory are also given. Thus, the quantum Monte Carlo method is of great use even for investigating dynamical quantum fluctuations in nonequilibrium systems.

I believe that the present book will be useful to active researchers in the frontier of condensed matter physics as well as to young graduate students who want to start learning the quantum Monte Carlo methods.

I would like to thank Dr. Dorota Lipowska for critical reading of some manuscripts by Japanese authors and also thank Dr. N. Hatano for his assistance in preparing the $\mathrm{LT}_{\mathrm{E}} \mathrm{X}$ style file.

Masuo Suzuki

Tokyo, September 1999 


\section{CONTENTS}

Preface

M. Suzuki

General Decomposition Theory of Exponential Operators

M. Suzuki

Quantum Monte Carlo and Related Methods - Recent Developments

N. Hatano and M. Suzuki

Monte Carlo Renormalization Group Study of the D=1 XXZ Model

M. A. Novotny and H. G. Evertz

Overcoming Critical Slowing Down in Quantum Monte Carlo Simulations

H. G. Evertz and M. Marcu

Quantum Manybody Spin Systems in Random Fields and Anisotropies

P. Reed

Inhomogeneity Effects in Quantum Spin Systems

S. Miyashita, J. Behre and S. Yamamoto

The Quantum Transfer Matrix and Its Application to Quantum Spin Chains

113 K. Kubo

Transfer Matrices in Quantum Many-Body Systems

T. Koma

Monte Carlo Calculations of Elementary Excitation

M. Takahashi

The Decoupled Cell Method of Quantum Monte Carlo Calculation

S. Homma

Decoupled Cell Monte Carlo Study of the Critical Properties of the

Spin-1/2 Ferromagnetic Heisenberg Model in Three Dimensions

R. J. Creswick and C. J. Sisson 
Variational Monte Carlo Studies of Correlated Electrons

H. Shiba

Quantum Monte Carlo Simulation of Multiband Fermion Systems

and its Application to Superconductivity

K. Kuroki and $\mathrm{H}$. Aoki

Quantum Monte Carlo in the Infinite Dimensional Limit

221

M. Jarrell, H. Akhlaghpour and Th. Pruschke

Aspects of the Sign Problem

J. H. Samson

Quantum Simulations of the Degenerate Single-Impurity Anderson Model

251

J. Bonća and J. E. Gubernatis

Quantum Monte Carlo Simulation by Auxiliary Fields

265

S. Sorella

Ground-State Projection Using Auxiliary Fields

S. Fahy

Fermion Simulations of Correlated Systems

299

M. Imada

Dirty Bosons in 2D: Phases and Phase Transitions

317

N. Trivedi and M. Makivić

Path-Integral Quantum Monte Carlo Studies of the Static and Time-

Dependent Thermodynamics of the Vibrational Properties of Crystals

A. R. MeGurn

Relaxation of Quantum Systems in Fluctuating Media

M. Takasu 